\title{
Reuse of syringes for therapeutic injections in Pakistan: rethinking determinants
}

\author{
Adnan Khan, ${ }^{1}$ Arshad Altaf, ${ }^{3}$ Huma Qureshi, ${ }^{2}$ Mejzgaan Orakzai ${ }^{1}$ and Ayesha Khan ${ }^{4}$
}

${ }^{1}$ Research and Development Solutions, Islamabad, Pakistan (Correspondence to: A.A. Khan: adnan@resdev.org). ${ }^{2}$ Pakistan Health Research Council, Islamabad, Pakistan. ${ }^{3}$ Service Delivery and Safety Department, World Health Organization, Geneva, Switzerland. ${ }^{4}$ Akhter Hameed Khan Resource Center, Islamabad, Pakistan.

\begin{abstract}
Background: Frequent reuse of syringes during medical injections is fuelling epidemics of human immunodeficiency virus and hepatitis $C$ virus infections in many low- and middle-income countries including Pakistan.

Aims: To explore specific factors related to syringe reuse during therapeutic injections.

Methods: We randomly surveyed 319 healthcare providers from 2 socioeconomically diverse districts in Pakistan, along with 625 of their patients.

Results: Providers see 12-25 patients per day, and provide 7-14 therapeutic injections or intravenous drips. Comparing daily stocks with injections provided, we estimated that 38\% of providers (Rawalpindi: 14\%, Tando Allah Yar: $44 \%$ ) likely reuse syringes 2 or 3 times. Rural location and longer duration of practice predict a higher likelihood of reuse. Physicians and non-physicians were equally likely to reuse. Most patients were unaware when a syringe had been reused.

Conclusions: High rate of syringe reuse is driven by high injection demand by patients, to which providers comply. Patients are generally unaware of the harm of injections with syringe reuse or that reuse happens. Our findings suggest that patient focused approaches may help reduce syringe reuse.

Keywords: injection demand, injection safety, syringe reuse, therapeutic injections, unsafe injections

Citation: Khan AA; Altaf A; Qureshi H; Orakzai M; Khan A. Reuse of syringes for therapeutic injections in Pakistan: rethinking its definition and determinants. East Mediterr Health J. 2020;26(3):283-289. https://doi.org/10.26719/emhj.19.028

Received: 31/08/17; accepted: 28/06/18

Copyright (c) World Health Organization (WHO) 2020. Open Access. Some rights reserved. This work is available under the CC BY-NC-SA 3.0 IGO license (https://creativecommons.org/licenses/by-nc-sa/3.o/).
\end{abstract}

\section{Introduction}

Syringe reuse during therapeutic injections has contributed to the global epidemics of hepatitis $C$ virus $(1,2)$ and human immunodeficiency virus (HIV) (3-6) infection, and is well documented in high-income $(7,8)$ and low- and middle-income countries (9-11). In Pakistan, high reuse of syringes during therapeutic injections (13) has led to a national prevalence of hepatitis $C$ of $4.8 \%$ (12), with some districts as high as $12 \%$, and has contributed to at least one community outbreak of HIV infection (14). Therapeutic injections in Pakistan range from 4.2-4.6 injections per person annually (13), with $17-50 \%$ of these injections being given with reused syringes $(12,13,15,16)$. Currently, conventional disposable syringes are used in Pakistan. The Punjab Government introduced reuse-prevention (RUP) syringes in its health facilities in 2017 (17). The World Health Organization's injection safety guidelines recommend RUP for all injections and sharp-injury protection syringes, wherever feasible (18).

We have previously demonstrated that the total national supply of syringes in Pakistan is sufficient to meet the demand for the $\sim 1.1$ billion syringes used annually for immunization, diabetes, laboratory testing and drug administration in clinics or hospitals (13). Therefore reuse of syringes cannot be attributed merely to a national shortfall of syringes as had been previously thought. However, such national aggregates hide reuse by individual practitioners. The present study explored the extent and pattern of syringe reuse in Pakistan. We also explored a novel method to identify reuse to avoid providers' self-promotion and patients' recall biases, as well as paradigms behind injection demand and supply in communities.

\section{Methods}

Rawalpindi and Tando Allah Yar were identified in collaboration with the Pakistani Ministry of Health as districts exemplifying high and low human development indices (19), to understand injection reuse practices across the extremes of human development. Rawalpindi is a large metropolis with a large number of public and private, and primary, secondary and tertiary medical care centres and specialists. Although around half of the population of Rawalpindi District is rural, the villages are close to the city. Tando Allah Yar is completely rural and poor. Data were collected between February and April 2009.

In the first phase, all healthcare providers from all 19 Union Councils of Tando Allah Yar and 38 (1: 5) randomly selected Union Councils from Rawalpindi were listed. Thus, 6053 providers were identified as any individuals who see patients in communities, irrespective of their training or licensure. This list was used to construct a simple randomization-sampling frame to recruit healthcare providers. The study was powered for type I 
error $(\alpha)$ : 0.05 and type II error $(\beta)$ : 0.2; giving a total of 320 providers to be divided between districts (Epi Info). The relative number of the providers between districts was weighted for the providers per Union Councils included in the listing, giving 174 providers from Rawalpindi and 145 from Tando Allah Yar. At each healthcare provider's facility, 2 patients were approached to participate in a brief survey, from among those present at the time of study team visit and based on who was encountered first.

Provider and patient questionnaires were pretested to ensure validity and appropriateness. The providers' questionnaire asked about their training and practice pattern. The patients' questionnaire asked about their demographics and healthcare-seeking behaviour. Both were asked about knowledge about syringe reuse and its harm.

Reuse of injections was identified by 3 measures: (1) the study teams observed providers while managing patients, including while giving injections; (2) recognizing that such observations may be subject to a Hawthorne effect $(20,21)$, where providers' behaviour may change when they recognize that they are being observed, we also asked patients if they felt that the injection they received was given with a new or reused syringe; and (3) since many patients may not have known whether a syringe was reused, we also used a proxy measure. We asked providers how many syringes they stocked for the previous day and compared these against the number of injections they said they had provided yesterday. This difference identified providers that were sure to reuse syringes since they would have insufficient syringes for all the injections they gave. This established a minimum level of reuse since providers can potentially reuse even when they have insufficient syringes. Recall bias was limited by asking only about the previous day. Variables were compared using SPSS+ version 20. Proportions of those that gave injections, and reusers were compared using $\chi^{2}$ tests. Predictors of reuse of syringes were explored using linear regression with predictors described in Table 5. These factors included all that were considered relevant to syringe reuse.

The study was reviewed and approved by the Ethical Review Committee of Bridge Consultants Foundation, Karachi, Pakistan. Informed consent was obtained from all participants.

\section{Results}

We interviewed 319 providers (145 from Tando Allah Yar and 174 from Rawalpindi). Their mean (standard deviation) age was 44.5 (11.8) years in Rawalpindi and 39.6 (10.2) years in Tando Allah Yar, and there were 265 (83\%) men. Around half $(57 \%)$ of all providers from Rawalpindi and $26 \%$ from Tando Allah Yar were physicians, with more urban than rural providers being physicians (Table 1). Urban providers from Rawalpindi saw a mean $27.5 \mathrm{pa}-$ tients a day and their rural counterparts saw a mean 16.7. Rural and urban providers from Tando Allah Yar saw a mean 19.9 and 21.8 patients a day respectively.

We interviewed 625 patients (273 from Tando Allah

\begin{tabular}{|c|c|c|c|c|}
\hline & Total & Rawalpindi & $\begin{array}{c}\text { Tando } \\
\text { Allah Yar }\end{array}$ & $\boldsymbol{P}$ \\
\hline \multicolumn{5}{|c|}{ Age of patients, yr, mean (SD) } \\
\hline All & $42.3(11.4)$ & $44.5(11.8)$ & $39.6(10.2)$ & $<0.001$ \\
\hline Rural & $39.5(10.7)$ & $42.9(11.5)$ & $37.2(9.5)$ & 0.002 \\
\hline Urban & $44.7(11.4)$ & 45.4 (11.9) & $43.4(10.4)$ & 0.271 \\
\hline \multicolumn{5}{|c|}{ Male sex, $n$ (\%) } \\
\hline All & $265(83 \%)$ & $133(77 \%)$ & $132(91 \%)$ & 0.001 \\
\hline Rural & $133(88 \%)$ & $52(83 \%)$ & $8191 \%)$ & 0.029 \\
\hline Urban & $132(80 \%)$ & $81(74 \%)$ & $51(91 \%)$ & $<0.001$ \\
\hline \multicolumn{5}{|c|}{ Physician (vs. non-physician), $n$ (\%) } \\
\hline All & $135(43 \%)$ & $98(57 \%)$ & $37(26 \%)$ & 0.003 \\
\hline Rural & $33(22 \%)$ & $18(29 \%)$ & $15(17 \%)$ & 0.015 \\
\hline Urban & $102(61 \%)$ & $80(73 \%)$ & $22(39 \%)$ & 0.003 \\
\hline \multicolumn{5}{|c|}{ Patients seen daily, mean (SD) } \\
\hline All & $22.4(18.6)$ & $23.8(20.5)$ & $20.6(15.9)$ & $<0.001$ \\
\hline Rural & $18.6(16.8)$ & $16.7(17.7)$ & $19.9(16.0)$ & $<0.001$ \\
\hline Urban & 25.7 (19.6) & $27.5(20.9)$ & $21.8(15.7)$ & 0.002 \\
\hline
\end{tabular}

Yar and 352 from Rawalpindi). Their mean age was 34.4 (18.7) years in Rawalpindi and 32.2 (12.1) years in Tando Allah Yar; 603 (56\%) were men (Table 2). Twenty percent of patients from Rawalpindi had no schooling, compared with 56\% from Tando Allah Yar. Patients from Tando Allah Yar were more likely to be farmers, labourers or housewives, while those from Rawalpindi were mostly skilled labourers, housewives, office workers or students

Commonest reasons for medical visits were fever, influenza-like symptoms or body aches (51\% of all patient visits) or abdominal symptoms such as pain, vomiting or diarrhoea (11\% of visits). An injection was provided during $53 \%$ of patient visits in Rawalpindi and $92 \%$ in Tando Allah Yar (Table 2). Patients from Rawalpindi reported having received a mean of 5.4 injections during the previous year compared to 13.2 injections by patients from Tando Allah Yar.

Patients from Tando Allah Yar reported a mean 3.8 visits to a healthcare provider by a member of their household during the previous month, compared to 2.5 by those from Rawalpindi (Table 2). During all such visits, an injection was given. Overall, $56 \%$ patients felt that an injection was necessary. Such perceptions were higher in Tando Allah Yar than in Rawalpindi (79\% vs. 39\%) (Table 2). Providers reciprocated such perceptions in that $44^{-}$ $56 \%$ of providers felt that an injection was required for common ailments such as fever, influenza, body aches or diarrhoea (Table 4). In practice, it was highly likely that an injection would be given for fever (OR: 7.9, $P=0.022$ ) but not for abdominal pain/ diarrhoea (OR 5.4, $P=0.187$ ).

Providers from Rawalpindi charged a mean US\$ 1.44 for a visit when no injection was given and US\$ 1.51 if an injection was given; there were no rural/urban differences. Providers from Tando Allah Yar charged a mean US\$ 0.59 


\begin{tabular}{|c|c|c|c|c|}
\hline Characteristics & $\begin{array}{c}\text { Total } \\
\text { Mean (SD) }\end{array}$ & $\begin{array}{l}\text { Rawalpindi } \\
\text { Mean (SD) }\end{array}$ & $\begin{array}{c}\text { Tando Allah Yar } \\
\text { Mean (SD) }\end{array}$ & $\boldsymbol{P}$ \\
\hline Age & $33.5(16.2)$ & $34.4(18.7)$ & $32.2(12.1)$ & 0.047 \\
\hline Male sex, $\mathrm{n}(\%)$ & $340(54 \%)$ & $178(50 \%)$ & $162(59 \%)$ & 0.588 \\
\hline \multicolumn{5}{|l|}{ Education } \\
\hline No schooling, n (\%) & $224(36 \%)$ & $72(20 \%)$ & $152(56 \%)$ & $<0.001$ \\
\hline Years of education, mean (SD) & $4.7(4.830)$ & $6.4(4.663)$ & $2.0(3.756)$ & $<0.001$ \\
\hline Was an injection prescribed during this visit, $\mathrm{n}(\%)$ & $614(70 \%)$ & $347(53 \%)$ & $267(92 \%)$ & $<0.001$ \\
\hline \multicolumn{5}{|l|}{ Injections received last year, mean (SD) } \\
\hline All & $8.2(13.5)$ & $5.4(9.7)$ & $13.2(17.4)$ & $<0.001$ \\
\hline Rural & $10.7(17.0)$ & $6.4(10.2)$ & $15.8(21.4)$ & $<0.001$ \\
\hline Urban & $6.4(9.9)$ & $4.9(9.4)$ & $10.1(10.3)$ & $<0.001$ \\
\hline \multicolumn{5}{|l|}{ Healthcare visits last month by a family member, mean (SD) } \\
\hline All & $3.1(4.6)$ & $2.5(5.7)$ & $3.8(2.5)$ & $<0.001$ \\
\hline Rural & $3.2(2.5)$ & $2.6(7.6)$ & $3.6(2.5)$ & 0.003 \\
\hline Urban & $3.1(4.6)$ & $2.5(4.5)$ & $4.0(2.4)$ & $<0.001$ \\
\hline \multicolumn{5}{|l|}{ Median injections received in these visits (SD) } \\
\hline All & $2(5.09)$ & $1.8(5.1)$ & $5.0(6.5)$ & $<0.001$ \\
\hline Rural & $3(6.65)$ & $2.7(7.8)$ & $6.2(7.8)$ & $<0.001$ \\
\hline Urban & $1(2.80)$ & $1.3(2.6)$ & $3.4(2.7)$ & $<0.001$ \\
\hline \multicolumn{5}{|l|}{ Felt an injection was necessary, $n$ (\%) } \\
\hline Yes & $351(56 \%)$ & $136(39 \%)$ & $215(79 \%)$ & $<0.001$ \\
\hline No, but the provider insisted & $18(3 \%)$ & $17(5 \%)$ & $1(0.4 \%)$ & 0.840 \\
\hline \multicolumn{5}{|l|}{ Median provider fee for this visit (in USD), $n$ (SD) } \\
\hline All & $1.10(1.36)$ & $1.48(1.65)$ & $0.61(0.49)$ & $<0.001$ \\
\hline Rural & $0.95(1.27)$ & $1.46(1.69)$ & $0.56(0.57)$ & $<0.001$ \\
\hline Urban & $1.24(1.41)$ & $1.49(1.64)$ & $0.69(0.30)$ & $<0.001$ \\
\hline Injection prescribed & $1.42(1.81)$ & $1.51(1.87)$ & $0.59(0.62)$ & $<0.001$ \\
\hline Injection not prescribed & $0.97(1.08)$ & $1.44(1.42)$ & $0.62(0.48)$ & $<0.001$ \\
\hline
\end{tabular}

when an injection was provided and US\$ 0.62 when not provided. Providers charged a mean US\$ 0.56 in rural and US\$ 0.69 in urban locations. Providers from Rawalpindi reported giving a mean of 8.8 intramuscular injections, 3.5 intravenous injections and 2.3 intravenous drip daily (Table 3). Providers from Tando Allah Yar gave a mean 10.0 intramuscular injections, 3.9 intravenous injections and 1.4 intravenous drip daily. We asked providers how many syringes they had stocked for the day: 140 (48\%) providers from Rawalpindi and 122 (46\%) from Tando Allah Yar gave more injections than their daily stock of syringes. Therefore, they would have been likely to reuse syringes regularly. For analysis, these were labelled as likely reusers. No urban provider from Rawalpindi fell into this category, while urban and rural providers in Tando Allah Yar were similar (Table 3). Around 38\% of all providers were likely to reuse consistently (Table 4). These likely reusers gave a mean 14.4 injections daily compared to 12.3 by those who were less likely to reuse syringes. Since reusers stocked around 5 syringes a day, they would likely have reused syringes for 9 injections in any given day.
Of the variables used in the linear regression model (Table 5), only practicing in Tando Allah Yar (AOR 1.92, range 1.9-7.69) and a longer duration of practice (AOR $0.6 \%$ for each year in practice, range $0.1-1 \%$ ) increased the likelihood for reuse. Physicians were just as likely to reuse as non-physicians. Our teams observed patient encounters for any injection reuse and we also asked patients if they had observed syringe reuse. Both of these modes of inquiry identified reuse during $<5 \%$ of observations/visits. Nearly all providers, but few patients, were aware of the possibility of acquiring injection site injuries or infections such as hepatitis or HIV infection from reused syringes.

\section{Discussion}

We found that around half of the patients had received an injection during their current visit and that at least $38 \%$ of the providers were likely to reuse syringes during injections. Reuse happens just as often by physicians or non-physicians and is irrespective of sex of providers or the fee charged. Both providers and patients felt that 


\begin{tabular}{|c|c|c|c|c|}
\hline \multicolumn{5}{|l|}{ Table 3 Injection provision } \\
\hline Injection provision & $\begin{array}{c}\text { Total } \\
\text { Mean (SD) }\end{array}$ & $\begin{array}{l}\text { Rawalpindi } \\
\text { Mean (SD) }\end{array}$ & $\begin{array}{c}\text { Tando Allah Yar } \\
\text { Mean (SD) }\end{array}$ & $\mathbf{P}$ \\
\hline \multicolumn{5}{|l|}{ Intramuscular injections provided daily } \\
\hline All & $9.4(9.6)$ & $8.8(9.6)$ & $10.0(9.6)$ & 0.134 \\
\hline Rural & $9.8(9.3)$ & $8.2(7.6)$ & $10.8(10.0)$ & 0.020 \\
\hline Urban & $9.1(9.8)$ & $9.2(10.4)$ & $8.9(8.7)$ & 0.819 \\
\hline \multicolumn{5}{|l|}{ Intravenous injections provided daily } \\
\hline All & $3.7(4.5)$ & $3.5(3.7)$ & $3.9(4.9)$ & 0.298 \\
\hline Rural & $3.6(4.2)$ & $2.8(3.4)$ & $3.9(4.4)$ & 0.057 \\
\hline Urban & $3.7(4.7)$ & $3.8(3.8)$ & $3.9(5.6)$ & 0.938 \\
\hline \multicolumn{5}{|l|}{ Intravenous drips provided daily } \\
\hline All & $1.7(3.8)$ & $2.3(3.9)$ & $1.4(3.8)$ & 0.037 \\
\hline Rural & $1.6(3.3)$ & & $1.0(1.6)$ & 0.003 \\
\hline Urban & $1.9(4.4)$ & $1.6(1.5)$ & $2.1(5.7)$ & 0.370 \\
\hline \multicolumn{5}{|l|}{ Injections of any kind daily } \\
\hline All & $15.7(13.4)$ & $17.6(15.6)$ & $14.8(12.6)$ & 0.083 \\
\hline Rural & $15.4(11.9)$ & $17.1(14.5)$ & $14.8(10.9)$ & 0.302 \\
\hline Urban & $16.0(15.1)$ & $18.1(15.6)$ & $14.8(14.9)$ & 0.193 \\
\hline Providers that are most likely to reuse syringes daily & $n(\%)$ & $n(\%)$ & $n(\%)$ & $n(\%)$ \\
\hline All & $262(47 \%)$ & $140(48 \%)$ & $122(46 \%)$ & 0.637 \\
\hline Rural & $127(49 \%)$ & $49(52 \%)$ & $78(48 \%)$ & 0.538 \\
\hline Urban & $135(45 \%)$ & $91(46 \%)$ & $44(44 \%)$ & 0.744 \\
\hline
\end{tabular}

\begin{tabular}{|c|c|c|c|}
\hline & Likely non-reusers & Likely reusers & $\boldsymbol{P}$ \\
\hline Likely reusers & $62 \%$ & $38 \%$ & \\
\hline Age, yr, mean (SD) & $38(10.5)$ & 40 (10.9) & 0.385 \\
\hline \multicolumn{4}{|l|}{ Physician or other, $n$ (\%) } \\
\hline MB BS & $196(62 \%)$ & $120(38 \%)$ & $<0.001$ \\
\hline Non-MB BS & $98(41 \%)$ & $142(59 \%)$ & 0.009 \\
\hline \multicolumn{4}{|l|}{ Sex of provider, $n(\%)$} \\
\hline Male & $232(50 \%)$ & $228(50 \%)$ & 1.000 \\
\hline Female & $52(64 \%)$ & $29(36 \%)$ & 0.019 \\
\hline Years since last completed degree (SD) & $21(10.8)$ & $24(8.8)$ & 0.807 \\
\hline Years in practice (SD) & $10(18.0)$ & $10(9.5)$ & 0.204 \\
\hline \multicolumn{4}{|l|}{ District } \\
\hline Tando Allah Yar, n (\%) & $143(54 \%)$ & $122(46 \%)$ & 0.196 \\
\hline Rawalpindi, n (\%) & $151(52 \%)$ & $140(48 \%)$ & 0.489 \\
\hline \multicolumn{4}{|l|}{ Location } \\
\hline Urban, n (\%) & $163(55 \%)$ & $135(45 \%)$ & 0.088 \\
\hline Rural, n (\%) & $131(51 \%)$ & $127(49 \%)$ & 0.749 \\
\hline No. of patient examined yesterday, mean (SD) & $19.4(16.7)$ & $28.4(20.1)$ & $<0.001$ \\
\hline No. of injections given daily, mean (SD) & $12.3(9.8)$ & $14.4(8.9)$ & 0.055 \\
\hline Fee per visit, US\$ (SD) & $0.60(0.79)$ & $0.60(0.69)$ & 0.171 \\
\hline \multicolumn{4}{|l|}{ Do you think injection is necessary for, $\mathbf{n}(\%)$} \\
\hline Fever & $107(54 \%)$ & $91(46 \%)$ & 0.264 \\
\hline Influenza-like symptoms & $72(56 \%)$ & $56(44 \%)$ & 0.182 \\
\hline Body aches & $136(53 \%)$ & $122(47 \%)$ & 0.338 \\
\hline Diarrhoea & $160(54 \%)$ & $136(46 \%)$ & 0.172 \\
\hline
\end{tabular}




\begin{tabular}{|c|c|c|c|c|c|c|c|c|c|}
\hline & \multicolumn{2}{|c|}{$\begin{array}{l}\text { Unstandardized } \\
\text { coefficients }\end{array}$} & \multirow{2}{*}{$\begin{array}{c}\text { Standardized } \\
\text { coefficients } \\
\beta\end{array}$} & \multicolumn{2}{|c|}{$95 \%$ CI for B } & \multirow[t]{2}{*}{ Sig } & \multirow[t]{2}{*}{$\begin{array}{l}\operatorname{Exp}(\mathrm{B}) \\
(\mathrm{AOR})\end{array}$} & \multicolumn{2}{|c|}{$\begin{array}{l}95 \% \text { CI for } \\
\text { AOR }\end{array}$} \\
\hline & B & SE & & $\begin{array}{l}\text { Lower } \\
\text { Bound }\end{array}$ & $\begin{array}{l}\text { Upper } \\
\text { Bound }\end{array}$ & & & $\begin{array}{c}\text { Lower } \\
\text { bound } \\
\text { of } \\
\text { AOR }\end{array}$ & $\begin{array}{c}\text { Upper } \\
\text { bound } \\
\text { of } \\
\text { AOR }\end{array}$ \\
\hline (Constant) & 0.405 & 0.752 & & -1.110 & 1.919 & 0.593 & 1.499 & 0.330 & 6.816 \\
\hline Urban/rural & 0.086 & 0.188 & 0.075 & -0.292 & 0.464 & 0.649 & 1.090 & 0.747 & 1.591 \\
\hline District & -0.522 & 0.194 & -0.434 & -0.914 & -0.130 & 0.010 & 0.593 & 0.401 & 0.878 \\
\hline Age of doctor & 0.009 & 0.017 & 0.183 & -0.025 & 0.044 & 0.581 & 1.009 & 0.976 & 1.045 \\
\hline Sex of doctor & 0.035 & 0.059 & 0.078 & -0.085 & 0.155 & 0.558 & 1.036 & 0.919 & 1.168 \\
\hline Year since last degree was completed & -0.008 & 0.016 & -0.172 & -0.040 & 0.023 & 0.592 & 0.992 & 0.961 & 1.023 \\
\hline How long you have been practicing at this clinic & -0.006 & 0.003 & -0.255 & -0.011 & 0.000 & 0.043 & 0.994 & 0.989 & 1.000 \\
\hline No. of patients examined yesterday & -0.011 & 0.006 & -0.363 & -0.022 & 0.001 & 0.079 & 0.989 & 0.978 & 1.001 \\
\hline No. of injections prescribed & 0.089 & 0.056 & 1.621 & -0.025 & 0.203 & 0.123 & 1.093 & 0.975 & 1.224 \\
\hline No. of intramuscular injections given yesterday & -0.061 & 0.053 & -0.882 & -0.167 & 0.046 & 0.257 & 0.941 & 0.846 & 1.047 \\
\hline No. of intravenous injection given yesterday & -0.064 & 0.055 & -0.471 & -0.174 & 0.047 & 0.252 & 0.938 & 0.840 & 1.048 \\
\hline No. of intravenous drips given yesterday & 0.012 & 0.042 & 0.038 & -0.073 & 0.097 & 0.776 & 1.012 & 0.930 & 1.102 \\
\hline Reuse of injections observed & -0.027 & 0.219 & -0.015 & -0.470 & 0.415 & 0.902 & 0.973 & 0.625 & 1.514 \\
\hline Reuse reported by the patient & -0.087 & 0.273 & -0.044 & -0.638 & 0.464 & 0.751 & 0.917 & 0.528 & 1.590 \\
\hline Physician/prescriber fee & -0.001 & 0.001 & -0.058 & -0.003 & 0.002 & 0.663 & 0.999 & 0.997 & 1.002 \\
\hline
\end{tabular}

Values in bold are significant. $\mathrm{AOR}=$ adjusted odds ratio; $\mathrm{CI}=$ confidence interval; $\mathrm{SE}=$ standard error; Sig = significance

injections were necessary for common ailments.

The high injection demand and provision seen in our study were consistent with prior experience from Pakistan (22) or the surrounding region (13,23-27). Patients expect to receive injections for minor ailments such as fever or influenza-like symptoms and willingly pay for these, on the mistaken belief in the efficacy of injections to overcome common symptoms that eventually abate with time (10). Healthcare providers comply with such wishes and are convinced of the necessity of injections. This belief is common among providers irrespective of whether they are likely or not to reuse syringes.

Syringe reuse happens against a backdrop of frequent injections. Around $38 \%$ of injection providers procure too few syringes for the injections that they provide and will likely reuse consistently. They also see more patients and give more injections. They charge slightly less per visit than providers that do not reuse syringes; however, their fees remain largely the same whether or not they give an injection. These providers stock a median of 5 syringes and give 14 injections daily; meaning that each syringe is reused 2 or 3 times. Providers' knowledge of the potential harm of syringe reuse and their incentives to reuse also mean that approaches such as information provision or availability of autodisposable syringes will not work, unless these are the only type of syringes available. Additionally, simply demanding or making laws against reuse are not likely to succeed. However, since providers' savings from syringe reuse are hidden from the patients, there may be a potential role for a patient-focused approach by which patients are made more aware of syringe reuse and its harm (18).
Community approaches that reduce information asymmetry between providers and patients have been promising (28). One intervention in Tando Allah Yar improved patient awareness from $15 \%$ to $29 \%$ within 6 months (29). Other complementary approaches may be to brand as safe providers those that visibly do not reuse syringes. Another option would be to use positive deviance inquiry in communities to reduce injection demand and syringe reuse (30-32).

One limitation of our study was that because we compared the supply of syringes versus injections given, we could only estimate the minimum reuse by providers. In reality, a provider may reuse more often, although perhaps not by much, because they would then adjust their syringe procurement accordingly in the long run.

\section{Conclusion}

Our study highlights the high prevalence of syringe reuse during therapeutic injections in communities in Pakistan and suggests that patient-centred approaches (demand reduction and increased awareness of the harm of syringe reuse), but probably not provider-centred approaches, may help reduce syringe reuse. New research should explore why patients seek such unnecessary care and test behavioural approaches such as cognitive behavioural therapy, expectation management, or whether patients will pay for safe injections, to make medical practice and injections safer in poor communities.

Funding: None.

Competing interests: None declared. 


\section{Réutilisation des seringues pour injections thérapeutiques au Pakistan : réflexion sur ses déterminants}

\section{Résumé}

Contexte : La réutilisation fréquente des seringues lors des injections thérapeutiques alimente des épidémies d'infections par les virus de l'immunodéficience humaine et de l'hépatite $C$ dans de nombreux pays à revenu faible et intermédiaire, y compris le Pakistan.

Objectifs : Étudier les facteurs spécifiques liés à la réutilisation des seringues lors des injections thérapeutiques.

Méthodes : Nous avons interrogé 319 prestataires de soins, de façon aléatoire, dans deux districts du Pakistan présentant une diversité socio-économique, ainsi que 625 de leurs patients.

Résultats : Les prestataires voient de 12 à 25 patients et effectuent de 7 à 14 injections thérapeutiques ou perfusions intraveineuses goutte-à-goutte par jour. En comparant les stocks journaliers avec les injections réalisées, nous avons estimé que $38 \%$ des prestataires (Rawalpindi : $14 \%$, Tando Allah Yar : $44 \%$ ) réutilisent vraisemblablement les seringues deux ou trois fois. L'implantation rurale et l'ancienneté d'exercice laissent anticiper une plus grande probabilité de réutilisation. Les médecins et les autres membres du personnel soignant étaient aussi susceptibles de réutiliser les seringues. Lorsqu'une seringue était réutilisée, la plupart des patients n'en avaient pas conscience.

Conclusions : Le taux élevé de réutilisation des seringues est induit par une forte demande d'injections par les patients, à laquelle répondent les prestataires de soins. En général, les patients n’ont pas conscience de la dangerosité des injections effectuées avec des seringues réutilisées, ni même de la pratique de réutilisation. Les résultats de notre enquête indiquent que des approches centrées sur le patient pourraient aider à réduire la réutilisation des seringues.

$$
\begin{aligned}
& \text { إعادة استعحال المحاقن للحَقْن العِلاَجِيّ في باكستان: إعادة النظر في استعمال المحاقن وححدداته } \\
& \text { عدنان خان، أرشد ألطف، هو ما قرشي، مزدجان أركازي، عايشة خان }
\end{aligned}
$$

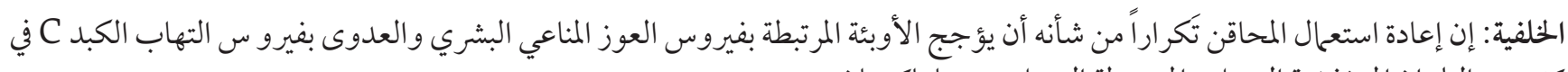

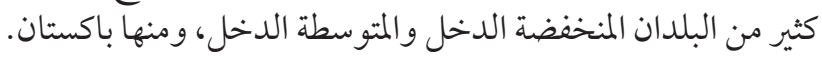
الأهداف: هدفت الدراسة إلى استكشاف العو امل المُحددة المرتبطة بإعادة استعمال المحاقن للحَقْن العِلاجِيّ

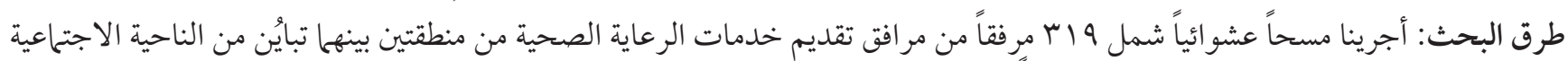

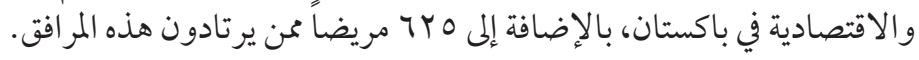

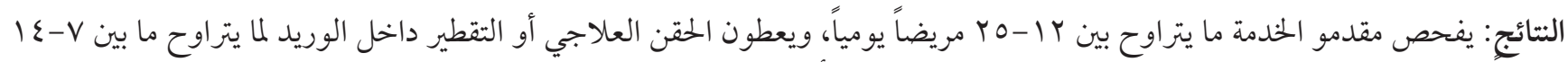

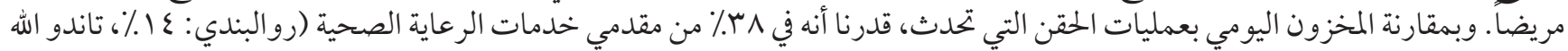

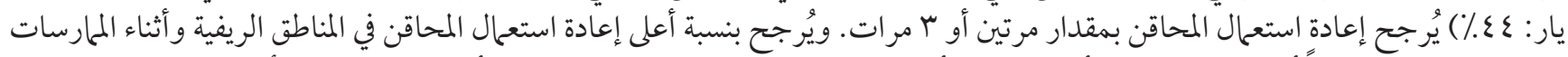

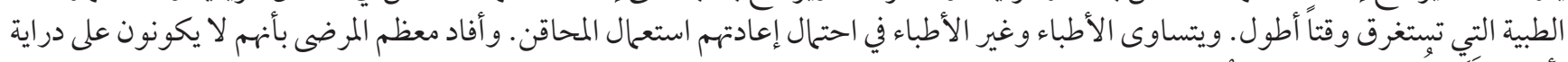

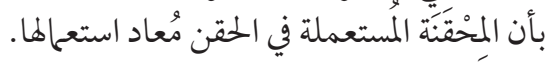

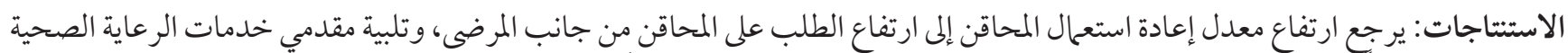

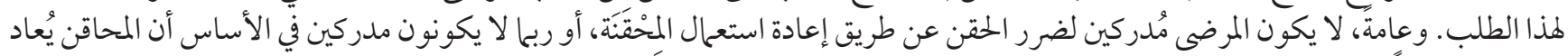

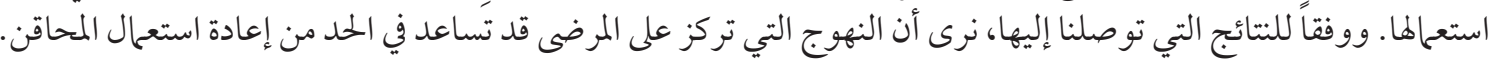

\section{References}

1. Brewer DD, Khan AA. HCV and HIV prevalences strongly correlated in Asian communities with reservoirs of HIV in high-risk groups. J Infect Dev Ctries. 2010 Aug 4;4(7):442-7. PMID:20818092

2. Shepard CW, Finelli L, Alter MJ. Global epidemiology of hepatitis C virus infection. Lancet Infect Dis. 2005 Sep;5(9):558-67. http://dx. doi.org/10.1016/S1473-3099(05)70216-4 PMID:16122679

3. Reid S, Van Niekerk AA. Injection risks and HIV transmission in the Republic of South Africa. Int J STD AIDS. 2009 Dec;20(12):816-9. http://dx.doi.org/10.1258/ijsa.2009.009230 PMID:19948894

4. Brewer DD, Gisselquist D, Brody S, Potterat JJ. Investigating iatrogenic HIV transmission in Ugandan children. J Acquir Immune Defic Syndr. 2007 Jun 1;45(2):253-4. http://dx.doi.org/10.1097/QAI.obo13e31805d8af9 PMID:17527097 
5. Potterat JJ, Brewer DD, Brody S. Miscarriage of HIV epidemiology in sub-Saharan Africa. AIDS. 2006 Apr 4;20(6):955-6. http://dx.doi. org/10.1097/01.aids.0000218571.35768.ce PMID:16549991

6. Brody S, Gisselquist D, Potterat JJ, Drucker E. Evidence of iatrogenic HIV transmission in children in South Africa. BJOG. 2003 May;110(5):450-2. PMID: 1274232

7. Comstock RD, Mallonee S, Fox JL, Moolenaar RL, Vogt TM, Perz JF, et al. A large nosocomial outbreak of hepatitis C and hepatitis B among patients receiving pain remediation treatments. Infect Control Hosp Epidemiol. 2004 Jul;25(7):576-83. http://dx.doi. org/10.1086/502442 PMID:15301030

8. Fischer GE, Schaefer MK, Labus BJ, Sands L, Rowley P, Azzam IA, et al. Hepatitis C virus infections from unsafe injection practices at an endoscopy clinic in Las Vegas, Nevada, 2007-2008. Clin Infect Dis. 2010 Aug 1;51(3):267-73. http://dx.doi.org/10.1086/653937 PMID:20575663

9. Simonsen L, Kane A, Lloyd J, Zaffran M, Kane M. Unsafe injections in the developing world and transmission of bloodborne pathogens: a review. Bull World Health Organ. 1999;77(10):789-800. PMID:10593026

10. Kermode M. Unsafe injections in low-income country health settings: need for injection safety promotion to prevent the spread of blood-borne viruses. Health Promot.Int. 2004 Mar;19(1):95-103. http://dx.doi.org/10.1093/heapro/dah11o PMID:14976177

11. Miller MA, Pisani E. The cost of unsafe injections. Bull World Health Organ. 1999;77(10):808-11. PMID:10593028

12. Qureshi H, Bile KM, Jooma R, Alam SE, Afridi HU. Prevalence of hepatitis B and C viral infections in Pakistan: findings of a national survey appealing for effective prevention and control measures. East Mediterr Health J. 2010;16 Suppl:S15-23. PMID: 2149558

13. Khan AA, Saleem M, Qureshi H, Jooma R, Khan A. Comparison of need and supply of syringes for therapeutic injections in Pakistan. J Pak Med Assoc. Nov 2012 Nov;62(11):1149-53. PMID:23866401

14. Ansari JA, Salman M, Safdar RM, Ikram N, Mahmood T, Zaheer HA, et al. HIV/AIDS outbreak investigation in Jalalpur Jattan (JPJ), Gujrat, Pakistan. J Epidemiol Glob Health. 2013 Dec;3(4):261-8. http://dx.doi.org/10.1016/j.jegh.2013.06.001 PMID:24206797

15. Khan AJ, Luby SP, Fikree F, Karim A, Obaid S, Dellawala S, et al. Unsafe injections and the transmission of hepatitis B and C in a periurban community in Pakistan. Bull World Health Organ. 2000 2000;78(8):956-63. PMID:10994278

16. Hutin YJ, Hauri AM, Armstrong GL. Use of injections in healthcare settings worldwide, 2000: literature review and regional estimates. BMJ. 2003 Nov 8;327(7423):1075. http://dx.doi.org/10.1136/bmj.327.7423.1075 PMID:14604927

17. Plan to introduce auto destructive syringes. Dawn. 17 November 2016 (https://www.dawn.com/news/1296822/plan-to-introduce-auto-destructive-syringes, accessed 15 May 2019).

18. WHO guideline on the use of safety-engineered syringes for intramuscular, intradermal and subcutaneous injections in health care settings. Geneva: World Health Organization; 2016 (http://apps.who.int/iris/bitstream/10665/250144/1/9789241549820-eng.pdf, accessed 1 October 2019).

19. List of districts of Pakistan by Human Development Index (https:/en.wikipedia.org/wiki/List_of_districts_of_Pakistan_by_Human_ Development_Index, accessed 15 May 2019).

20. McCambridge J, Witton J, Elbourne DR. Systematic review of the Hawthorne effect: new concepts are needed to study research participation effects. J Clin Epidemiol. 2014 Mar;67(3):267-77. http://dx.doi.org/10.1016/j.jclinepi.2013.08.015 PMID:24275499

21. McCarney R, Warner J, Iliffe S, van Haselen R, Griffin M, Fisher P. The Hawthorne Effect: a randomised, controlled trial. BMC Med Res Methodol. 2007 Jul 3;7:30. http://dx.doi.org/10.1186/1471-2288-7-30 PMID:17608932

22. Raglow GJ, Luby SP, Nabi N. Therapeutic injections in Pakistan: from the patients' perspective. Trop Med Int Health. 2001 Jan;6(1):6975. http://dx.doi.org/10.1046/j.1365-3156.2001.00653.x PMID:11263465

23. Anand K, Panday CS, Kapoor SK. Injection use in a village in north India. Natl Med J India. 2001 May-Jun;14(3):143-144. PMID:11467141

24. Gyawali S, Rathore DS, Shankar PR, Maskey M, Vikash KK. Injection practices in Nepal: health policymakers' perceptions. BMC Int Health Hum Rights. 2014 Jun 24;14:21. http://dx.doi.org/10.1186/1472-698X-14-21 PMID:24957575

25. Altaf A, Janjua NZ, Hutin Y. The cost of unsafe injections in pakistan and challenges for prevention program. J Coll Physicians Surg Pak. 2006 Sep;16(9):622-4. http://dx.doi.org/9.2006/JCPSP.622624 PMID:16945242

26. Bhunia R, Hutin Y, Ramkrishnan R, Ghosh PK, Dey S, Murhekar M. Reducing use of injections through interactional group discussions: a randomized controlled trial. Indian Pediatr. 2010 May;47(5):409-14. http://dx.doi.org/10.1007/s13312-010-0076-4 PMID:19736370

27. Kermode M, Muani V. Injection practices in the formal \& informal healthcare sectors in rural north India. Indian J Med Res. 2006 Nov;124(5):513-20. PMID:17213519

28. Kermode M, Holmes W, Langkham B, Thomas MS, Gifford S. Safer injections, fewer infections: injection safety in rural north India. Trop Med Int Health. 2005 May;10(5):423-32. http://dx.doi.org/10.1111/j.1365-3156.2005.01421.x PMID:15860088

29. Altaf A, Shah SA, Shaikh K, Constable FM, Khamassi S. Lessons learned from a community based intervention to improve injection safety in Pakistan. BMC Res Notes. 2013 Apr 22;6:159. http://dx.doi.org/10.1186/1756-0500-6-159 PMID:23607289

30. Friedman SR, Mateu-Gelabert P, Sandoval M, Hagan H, Des J, D.C. Positive deviance control-case life history: a method to develop grounded hypotheses about successful long-term avoidance of infection. BMC Public Health. 2008 Mar 20;8:94.

31. Marsh DR, Schroeder DG, Dearden KA, Sternin J, Sternin M. The power of positive deviance. BMJ. 2004 Nov13;329(7475):1177-9. http:// dx.doi.org/10.1136/bmj.329.7475.1177 PMID:15539680

32. Lapping K, Marsh DR, Rosenbaum J, Swedberg E, Sternin J, Sternin M, et al. The positive deviance approach: challenges and opportunities for the future. Food Nutr Bull. 2002 Dec;23(4 Suppl):130-7. PMID:12503241 